The Egyptian Journal of Hospital Medicine (July 2020) Vol. 80 (2), Page 943-950

\title{
Prediction of Acute Kidney Injury in Critically-Ill Pediatric Patients Admitted to PICU: The Role of Serum Cystatin C And Serum Interleukin-18 Hams Ahmed Attalla ${ }^{*}$, Ali Mahmoud Ahmed ${ }^{2}$ \\ ${ }^{1}$ Department of Pediatrics, Faculty of Medicine, Helwan University, ${ }^{2}$ Department of Neurology, Faculty of Medicine, Al-Azhar University, Cairo, Egypt. *Correspondence to Dr. Hams Ahmed Attalla; Email: hamsattala20002003@yahoo.com.
}

\section{ABSTRACT}

Objectives: We conducted this study to assess the sensitivity and specificity of serum cystatin C (sCysC) and serum interleukin-18 (IL-18) in comparison with serum creatinine and serum urea for early detection of acute kidney injury (AKI) in critically-ill pediatric patients admitted to the pediatric intensive care unit (PICU).

Methods: This was an observational case-control study enrolled 61 patients admitted in PICU divided into two groups (the case group 30 patients with proposed AKI and the control group 31 control individuals). After complete clinical evaluation, electrolyte profile, including $\mathrm{Na}$ and $\mathrm{K}$ besides the biomarkers assay of serum creatinine, urea, sCysC , and IL-18 were measured at the first day of admission and after three days.

Results: Urea and creatinine were increased at admission in only three patients and two patients, respectively, while it dramatically increased in 25 patients in both urea and creatinine separately on the third day of admission. sCysC and sIL-18 showed a high diagnostic ability for detecting AKI at admission with AUCs of 0.877 and 0.819 respectively. Supportingnly, sCysC and IL-18 were significantly higher in AKI group compared to the control group. Conclusions: $\mathrm{sCysC}$ and serum IL-18 proved their accuracy in prediction of AKI in patients admitted to the PICU. Their diagnostic ability was superior to serum creatinine, serum urea, and even urinary IL-18 and CysC. Keywords: Serum Cystatin C; Serum Interleukin-18; Acute Kidney Injury; PICU.

\section{INTRODUCTION}

As an intense and permanent deterioration of the kidney functions leads to accumulation of waste products and metabolic disturbances arrives in developing of acute kidney injury (AKI) that had a higher incidence of morbidity and mortality especially in critically-ill patients due to sepsis, convulsions and uremia $^{(1}$. Of date, the burden of AKI is about $27 \%$ of children admitted to the pediatric intensive care unit (PICU) with a mortality rate approximately $10 \%$, whilst neonatal incidence widely varied from $8 \%$ to $24 \%$ with mortality rate two to even five patients every ten patients admitted to NICU (2). In spite of the prerenal causes, as sepsis, hypovolemia, hypoxia and congenital urological abnormalities, were considered the commonest, ${ }^{(2)}$ AKI may be developed as exacerbation of chronic kidney disease (CKD) ${ }^{(3)}$.

Early diagnosis and proper management of AKI is the magnificent purpose as regard as AKI is reversible if the proper decision was taken at the adequate time. Otherwise, the kidney is a reticence organ so, deterioration of its functions took time to be manifested clinically so that the biomarkers is the key to early detection of renal dysfunction ${ }^{(3)}$.

Among the past era, serum creatinine, urea, and urine output were the standard parameters for the diagnosis of AKI as monitors of the glomerular filtration rate and excretory function of the kidney. However, urine output is of limited value as it is affected by previous body hydration state and fluid intake $^{(4)}$. Having serum creatinine, it is not appropriate predictor for early changes in renal function as it is affected by the patient's sex, age, metabolic state, dehydration, drugs and muscle bulk. Interestingly, throughout the first three days of neonatal period serum creatinine reflects the maternal levels rather than the neonatal levels (2). Moreover, serum creatinine concentration may be stationary in spite of half function of the kidney was lost ${ }^{(5)}$. Thus, serum creatinine may respite diagnosis, management, and prohibition of AKI sequel.

The limited value of serum creatinine in AKI brings to light the need for new potential biomarkers with higher accuracy and ability to detect even subclinical AKI simplifying early diagnosis, evident intervention and assessment of the progression of the disease.

Serum interleukin-18 (IL-18) and serum cystatin $\mathrm{C}$ (sCysC) are potential biomarkers, proved their abilities not only in the early detection of physiological and pathological derangements accompaning AKI but also the prognosis of the disease ${ }^{(6)}$. Interleukin-18 is one of IL-1 pro-inflammatory cytokine family that is produced in an inactive form inside proximal convoluted tubules (PCT) then activated to $18.3 \mathrm{kDa}$ cytokine ${ }^{(7)}$. Furthermore, it was proved that IL-18 has a significant role in the early prediction of renal ischemia and reperfusion injury. It is increased after six

This article is an open access article distributed under the terms and conditions of the Creative Commons Attribution (CC BY-SA) license (http://creativecommons.org/licenses/by/4.0/) 
hours and reaching its peak within 12 hours with sensitivity two days earlier than serum creatinine level (8). Otherwise, interleukin-18 proved specificity more than $90 \%$ as an early mediator of AKI and its level is not affected by UTI, nephritic syndrome or even CKD (9).

On the other hand, sCysC, an intrinsic protease inhibitor that acts as an indicator of GFR, was not reported to be affected by age, gender, and muscle state. This elucidate the accuracy and sensitivity for the early detection of AKI two days earlier than serum creatinine ${ }^{(10)}$. Therefore, it can act as a strong predictor of mortality among patients with AKI ${ }^{(3)}$. In the neonatal period, sCysC reflects the actual state of the kidney filtration as it cannot pass the placenta ${ }^{(11)}$. On the other hand, sCysC level may be affected by thyroid state, steroid intake, lipid profile, inflammations, and malignancy.

In spite that studies reported the role of IL-18 and sCysC in the early detection and prognosis of $\mathrm{AKI}$, the literature is limited especially in critically-ill pediatric patients. Subsequently, we conducted this study to assess the sensitivity and specificity of serum CysC and serum IL-18 in comparison with serum creatinine and serum urea for the early detection of AKI in criticallyill pediatric patients admitted to the PICU.

\section{MATERIAL AND METHODS}

This was an observational case-control study executed at the Department of Pediatrics and Neonatology at El-Hussein University Hospital, Faculty of Medicine, Al-Azhar University, Cairo from January 2017 to December 2017.

\section{Ethical approval}

Research Ethics Board approval was gained from the Ethics Unite of the Faculty of Medicine, AlAzhar University. Furthermore, informed consents were obtained from parents or legal trustee of the included patients with receptiveness to the Declaration of Helsinki.

Study population: An overall 61 patients enrolled in our study were divided into two groups. Thirty patients were included in the case group, whilst thirty-one were enrolled in the control group.

Inclusion criteria: We included patients, aged from 90 days to 18 years, presented clinically with renal dysfunction with symptoms and signs like; puffiness of eyelids, generalized anasarca, elevated blood pressure, change in urine volume (oliguria or polyuria), confusion, and convulsion in severe cases. Each case was matched with a control patient without clinical evidence of AKI. Exclusion criteria: We excluded patients with a previously known renal disease, previous renal transplantation, previous administration of nephrotoxic agents, end-stage renal disease, recent cardiac surgery, patients admitted less than two days in PICU, multiple congenital anomalies, or multiorgan dysfunction.

\section{AKI definition in children}

Based on the modified pediatric RIFLE (pRIFLE) criteria that established the patients at risk of AKI when $25 \%$ decrease in estimated glomerular filtration rate (eGFR) and/or urine output $<0.5 \mathrm{ml} / \mathrm{kg} / \mathrm{h}$ for $8 \mathrm{~h}$. Subsequently, 50\% decrease in eGFR and/or urine output $<0.5 \mathrm{ml} / \mathrm{kg} / \mathrm{h}$ for $16 \mathrm{~h}$ elucidate that patients had renal injury, whilst renal failure was decided when $75 \%$ decrease in estimated GFR from baseline renal function and/or urine output $<0.3 \mathrm{ml} / \mathrm{kg} / \mathrm{h}$ for $24 \mathrm{~h}$. Eventually, persistent AKI more than four weeks delineated the patients with lost renal function. Schwartz formula was used to determine the estimated creatinine clearance $(\mathrm{eCCI})^{(12)}$.

\section{Clinical data collection}

All patients admitted at PICU for at least three days were subjected to meticulous history taking and clinical examination. The clinical examination included the vital signs, oedema, urine volume, signs of renal impairment, level of consciousness, and other systems. Collected clinical data included patients' age, sex, BMI, length of PICU stay and length of hospital stay. Moreover, associated co-morbidities implicated neurological, cardiac, respiratory, gastrointestinal and malignancy data were estimated.

\section{Urine collection and analysis}

Eligible patients enrolled in the study had urine collected from a lodging urinary catheter, collection bags or even cotton balls from patients wearing the diaper with an assessment of daily urine output every eight hours. Samples were stored in ice or in a $4^{\circ} \mathrm{C}$ refrigerator within four hours of collection and are stored until they are processed. The samples were examined during the first hour for assessment of the physical properties by inspecting and smelling. Furthermore, the dipping urine strips (Medi Test urine strips) were used for determination of the chemical characters after waiting for 30 seconds. The microscopic analysis was done by centrifuging the samples for 10 minutes at $3000 \mathrm{rpm}$. The supernatant fluids were emptied, and the deposit was examined on a glass slide and covered under the microscope. Eventually, urine output (UOP) and glomerular filtration rate (GFR) were assessed in the first 48 hours only.

\section{Biomarkers assessment}

The assays were conducted using the commercial ELISA technique. CysC and IL-18 standards were reconstituted by pouring $1.0 \mathrm{ml}$ of deionized water in the lyophilised standard gently and were let for 30 and 15 min, respectively. Subsequently, six serial calibrators $(100,50,25,12.5,6.25$ and $3.12 \mathrm{ng} / \mathrm{ml}$ respectively, by using diluted calibrator diluent) and 
six serial diluted standards $(500,250,125,62.5,31.2$, $15.6 \mathrm{pg} / \mathrm{ml}$, respectively, by using sample diluent) were done for sCysC and sIL-18, respectively. After incubation for two hours, the plates were shacked to homogenize the content. Reading was done by using ELISA STAST FAX reader adjusted $450 \mathrm{~nm}$. The concentrations were obtained by interpolation.

Serum creatine was measured using an IDMS traceable enzymatic assay. As regards samples were collected during the first clearance interval, they were stored at $80^{\circ} \mathrm{C}$. On the other hand, serum urea was assessed using blood urea nitrogen (BUN) test within one hour after venipunctures. All laboratory data of the included patients were subjected to quality assessment and biomarkers were assessed and personnel detection was blinded to clinical consequences.

\section{Statistical analysis}

To compare categorical paired variables McNemar's exact test was used. Whilst, for continuous outcomes, we used Wilcoxon sign rank test for nonnormally distributed data. Subsequently, Correlation analysis was performed using Pearson correlation coefficient for continuous normally-distributed variables, while the Spearmann rank correlation was used for other variables. Receiver operating characteristic (ROC) curve and area under ROC curve (AUC) were used to assess the diagnostic ability of biomarkers using MedCalc software version 14.8 (MedCalc Software, Mariakerke, Belgium). The optimal cut off points was calculated to maximize sensitivity and specificity. All tests were considered significant when $\mathrm{P} \leq 0.05$. Statistical analysis was performed using SPSS software version 22 for Windows (SPSS Inc., Chicago, IL, USA).

\section{RESULTS}

Thirty patients diagnosed with AKI were included with 31 matched control after admission to the PICU. AKI group had 18 (60\%) males and $12(40 \%)$ females while the control group had $20(64.5 \%)$ males and 11 (35.4\%) females. Moreover, the median age of the AKI group was 35 months (6-180), while the control group had higher age with 45 months (8-156). Furthermore, the median BMI was 22 in the AKI group and 20 in the controls. All general clinical and laboratory characteristics were assessed at admission and after three days. Despite the absence of difference between the two groups regarding the level of $\mathrm{sCr}$ at the day of admission, its level was significantly higher after 72 hours $(P<0.0001)$. Similarly, there was a significant difference between the two groups regarding the urea level at the third day. Interestingly, the levels of scysC and sIL-18 were significantly higher in the case group at the time of admission than in the control group $(P<$ $0.0001)$. In addition, the lengths of PICU stay and hospital stay were significantly higher in AKI patients than in non-AKI patients. There was no difference between the two groups regarding the other comorbidities (Table 1).

Table (1): Clinical and laboratory characteristics of included study patients

\begin{tabular}{|c|c|c|c|c|c|c|}
\hline & & \multicolumn{2}{|l|}{ AKI } & \multicolumn{2}{|c|}{ Non-AKI } & \multirow{3}{*}{$P$ value } \\
\hline & & Mean*/ & $\mathrm{SD} * /$ & Mean*/ & SD*/ & \\
\hline & & Median/Number & Range/\% & Median/Number & Range/\% & \\
\hline \multicolumn{2}{|c|}{ Age } & 35 & $6-180$ & 45 & $8-156$ & 0.295 \\
\hline \multicolumn{2}{|c|}{ Gender (Male) } & 18 & $60 \%$ & 20 & $64.5 \%$ & 0.864 \\
\hline \multicolumn{2}{|c|}{ BMI } & 22 & $16-29$ & 20 & $16-29$ & 0.705 \\
\hline \multirow{2}{*}{ Creatinine } & At admission & 0.71 & $0.2-1.6$ & 0.55 & $0.25-1.2$ & 0.06 \\
\hline & After $72 \mathrm{H}$ & 1.9 & $0.5-3.7$ & 0.62 & $0.28-1.1$ & $<0.0001$ \\
\hline \multirow{2}{*}{ Urea } & At admission & 14.2 & $8-42$ & 8.3 & $4.3-12.2$ & 0.03 \\
\hline & After $72 \mathrm{H}$ & 28.1 & $15-57$ & 6.1 & $4.2-10.6$ & $<0.0001$ \\
\hline \multicolumn{2}{|c|}{ Cystatin C } & 1.8 & $0.3-3.1$ & 0.28 & $0.05-2.9$ & $<0.0001$ \\
\hline \multicolumn{2}{|c|}{ IL-18 } & 343 & $128-528$ & 142 & $44-345$ & $<0.0001$ \\
\hline \multicolumn{2}{|c|}{ Length of PICU stay (d) } & $10.4^{*}$ & $3.1 *$ & $7.5^{*}$ & $2.4^{*}$ & $<0.0001$ \\
\hline \multicolumn{2}{|c|}{ Length of hospital stay (d) } & $29.4^{*}$ & $2.8^{*}$ & 25.9 & $3.6^{*}$ & $<0.0001$ \\
\hline \multicolumn{7}{|c|}{ Other diseases } \\
\hline \multicolumn{2}{|c|}{ Respiratory } & 6 & $20 \%$ & 8 & $25.8 \%$ & 0.182 \\
\hline \multicolumn{2}{|c|}{ Cardiac } & 3 & $10 \%$ & 5 & $16.1 \%$ & 0.717 \\
\hline \multicolumn{2}{|c|}{ Neurological } & 8 & $26.7 \%$ & 11 & $35.4 \%$ & 0.589 \\
\hline \multicolumn{2}{|c|}{ Malignancy } & - & - & 1 & $3.2 \%$ & - \\
\hline \multicolumn{2}{|c|}{ Gastrointestinal } & 3 & $10 \%$ & 4 & $12.9 \%$ & 0.983 \\
\hline
\end{tabular}

Data represented as mean $\pm \mathrm{SD}$, for continuous normally distributed data, Median and range, for continuous non-normally distributed data, along with number and percentage for categorical data.

AKI, Acute kidney injury; IL, Interleukin. 
Having the ROC curve and the AUC analysis, cysC showed a highly diagnostic ability for detecting AKI at admission with AUCs of 0.877 (95\% CI 0.768-947, P < 0.0001). Furthermore, AKI was highly detected also by using IL-18 with AUC of 0.819 (95\% CI 0.698-0.907, P < 0.0001). (Table 2, Fig 1-A and B). The optimal cutoff value was $0.8 \mathrm{mg} / \mathrm{L}$ for cysC and $212 \mathrm{Pg} / \mathrm{ml}$ for IL-18 (Table 3). Supportingnly, cystatin C and IL-18 were significantly higher in AKI group compared to the control group (Fig 2). Moreover, both biomarkers were correlated with creatinine and urea after 72 hours of admission (Table 4). Furthermore, creatinine and urea did not show high diagnostic performance on assessing AKI at admission with an AUC of 0.524 (95\% CI 0.299-0.749, P $=0.867)$ and 0.520 (95\% CI 0.245-0.795, P = 0.889), respectively (Table 2, Fig 1-C and D). Subsequently, creatinine and urea did not have any significant difference between AKI and control group at admission. Additionally, both biomarkers were significantly different in AKI group between admission level and the level after 72 Hours.

Both cystC and IL-18, were inversely correlated with age $(\mathrm{R}=-0.305, \mathrm{P}=0.017),(\mathrm{R}=-0.342, \mathrm{P}=0.007)$ (Fig 3-A and B). Moreover, BMI had a significant negative correlation with cystC $(\mathrm{R}=-0.299, \mathrm{P}=0.021)$. On the other hand, IL-18 was not correlated with BMI $((\mathrm{R}=-0.093, \mathrm{P}=0.480)($ Fig $3-\mathrm{C}$ and $\mathrm{D})$.

Table (2): Diagnostic ability of kidney biomarkers in acute kidney injury

\begin{tabular}{cccc}
\hline & AUC & $\mathbf{9 5 \%}$ CI & P value \\
\hline Cystatin C & 0.877 & $0.768-947$ & $<0.0001$ \\
\hline IL-18 & 0.819 & $0.698-0.907$ & $<0.0001$ \\
\hline Creatinine & 0.536 & $0.346-0.719$ & 0.806 \\
\hline Urea & 0.569 & $0.434-0.696$ & 0.362 \\
\hline
\end{tabular}

AUC, Area under curve; CI, Confidence interval.

Table (3): Diagnostic efficiency of kidney biomarkers in predicating AKI

\begin{tabular}{lccccccc} 
& Cut off value & Sensitivity & Specificity & PPV & NPV & PLR & NLR \\
\hline Cystatin C & 0.8 & $75 \%$ & $89.66 \%$ & $88.9 \%$ & $76.5 \%$ & 7.25 & 0.28 \\
\hline IL-18 & 212 & $65.6 \%$ & $89.3 \%$ & $87.5 \%$ & $69.4 \%$ & 6.13 & 0.39 \\
\hline Creatinine & 0.6 & $72 \%$ & $40 \%$ & $85.7 \%$ & $22.2 \%$ & 1.20 & 0.70 \\
\hline Urea & 12 & $53.1 \%$ & $64.3 \%$ & $63 \%$ & $54.5 \%$ & 1.49 & 0.73 \\
\hline
\end{tabular}

PPV, Positive predictive value; NPV, Negative predictive value; PLR, Positive likelihood ratio; NLP, Negative likelihood ratio.

Table (4): Correlation between kidney biomarkers in acute kidney injury

\section{IL-18 at admission Cystatin $\mathrm{C}$ at admission}

\begin{tabular}{ccccc}
\hline & Correlation coefficient & $\mathrm{P}$ value & Correlation coefficient & $\mathrm{P}$ value \\
\hline $\begin{array}{c}\text { Creatinine at } \\
\text { admission }\end{array}$ & 0.120 & 0.529 & 0.220 & 0.459 \\
\hline Creatinine after 3 days & 0.760 & $<0.001$ & 0.520 & 0.003 \\
\hline Urea at admission & 0.149 & 0.432 & 0.190 & 0.360 \\
\hline Urea after 3 Days & 0.520 & 0.003 & 0.280 & 0.134 \\
\hline
\end{tabular}



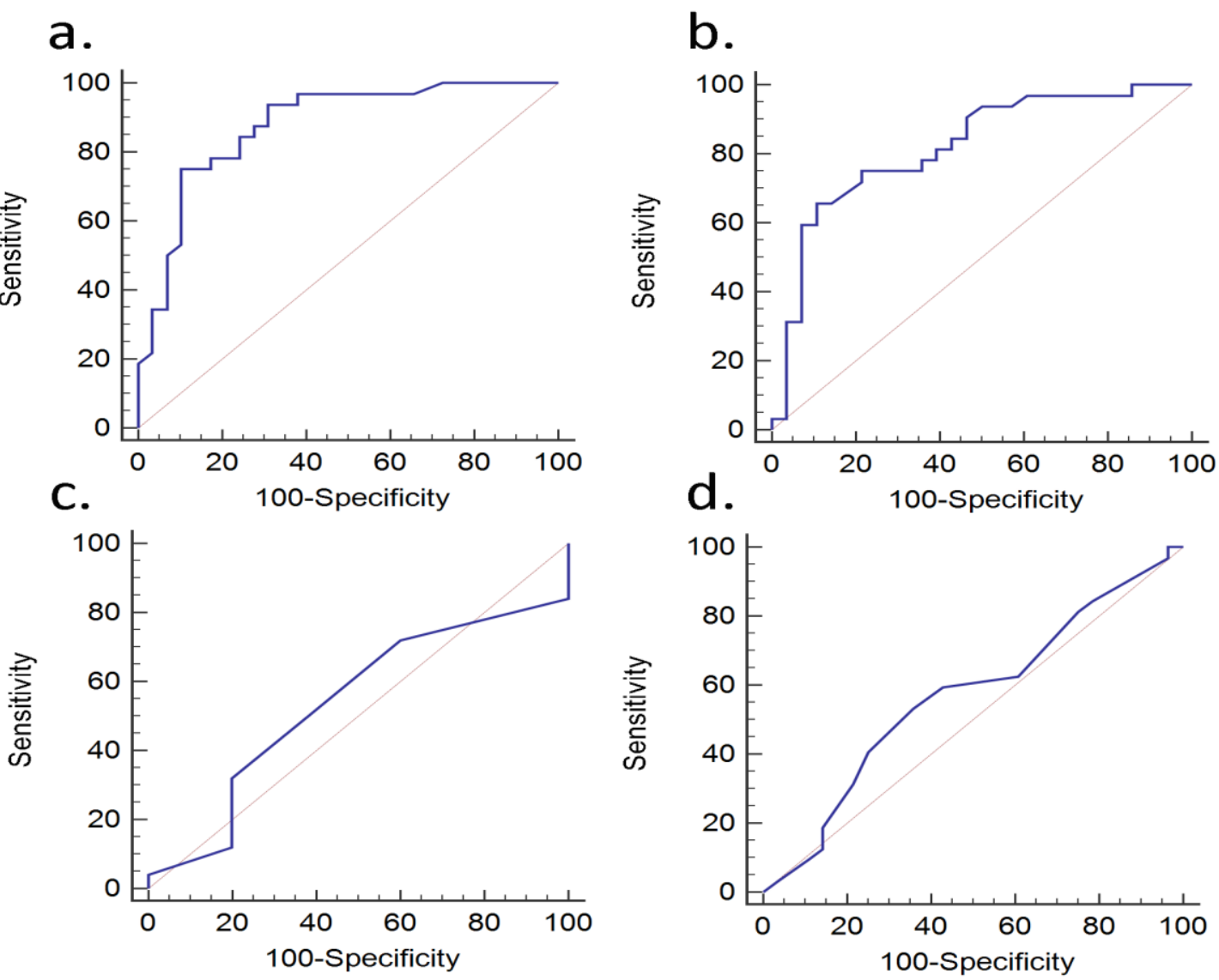

Figure (1): Receiver operating characteristic (ROC) curve for the diagnostic ability of serum renal biomarkers in predicting acute kidney injury (AKI) at admission. a; ROC curve for cystatin C, b; ROC curve for Inter 18 (IL18), $\mathbf{c} ;$ ROC curve for creatinine and $\mathbf{d}$ ROC curve for urea.

a.

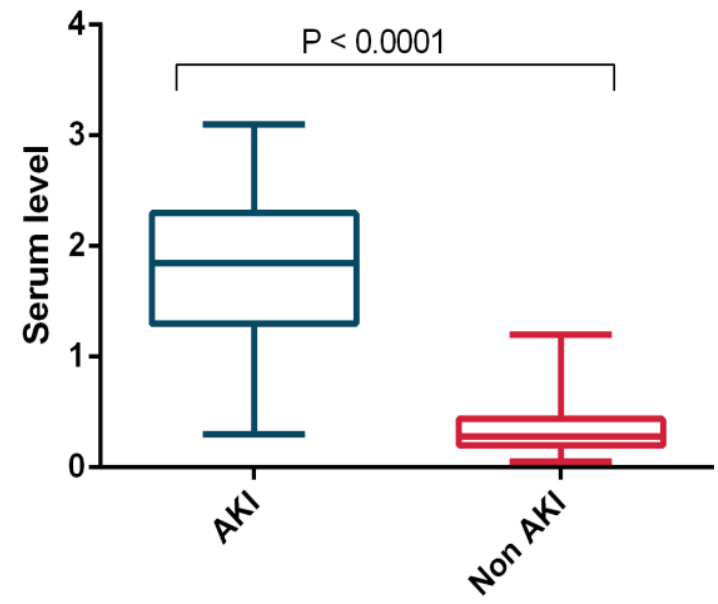

b.

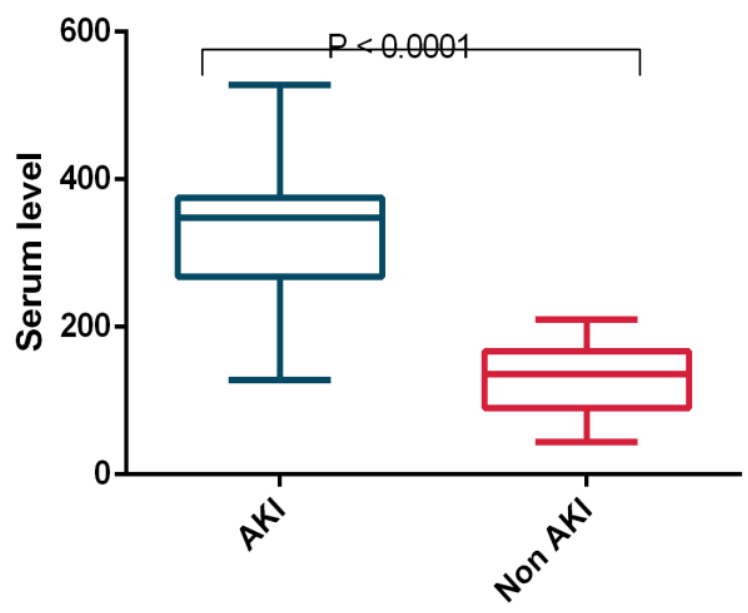

Figure (2): Box and whisker plot comparing renal biomarkers between acute kidney injury group (AKI) and nonAKI group. a; Cystatin C, b; Interleukin 18 (IL-18). 


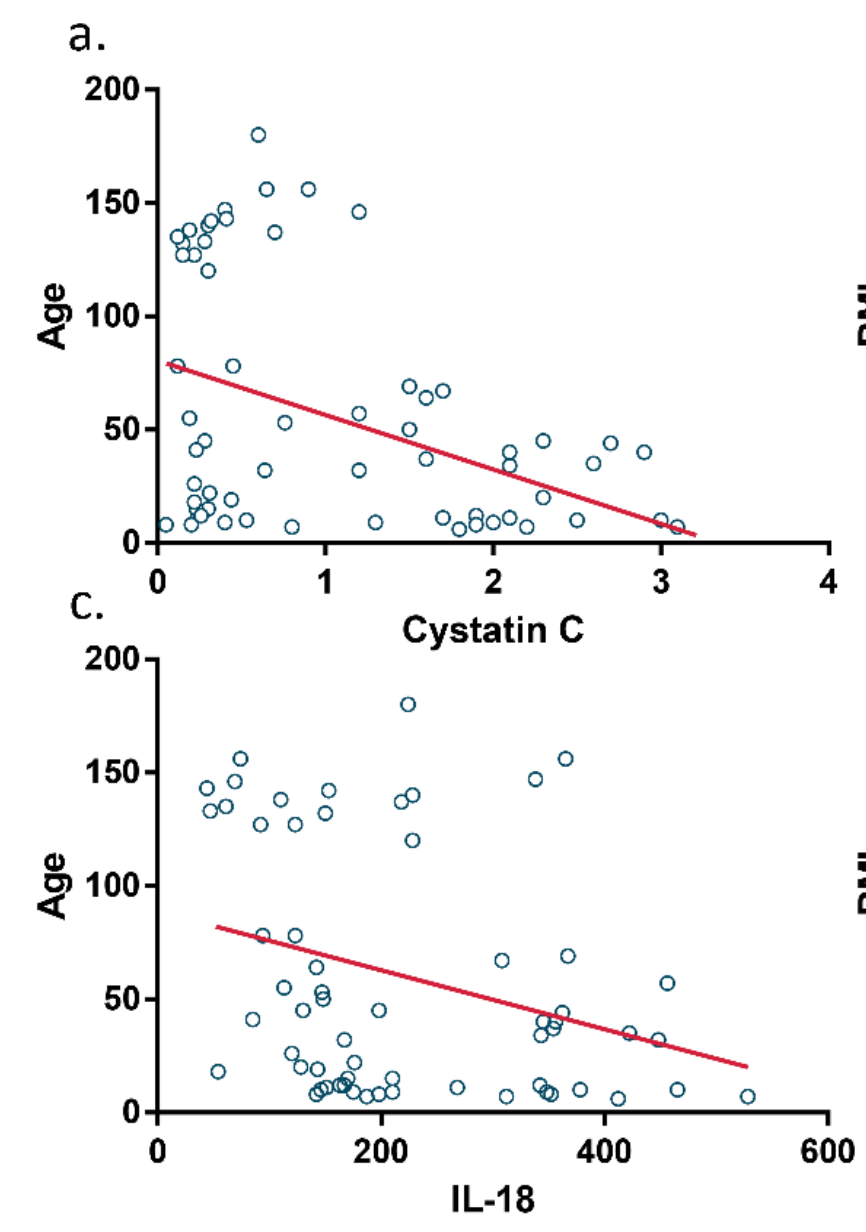

b.

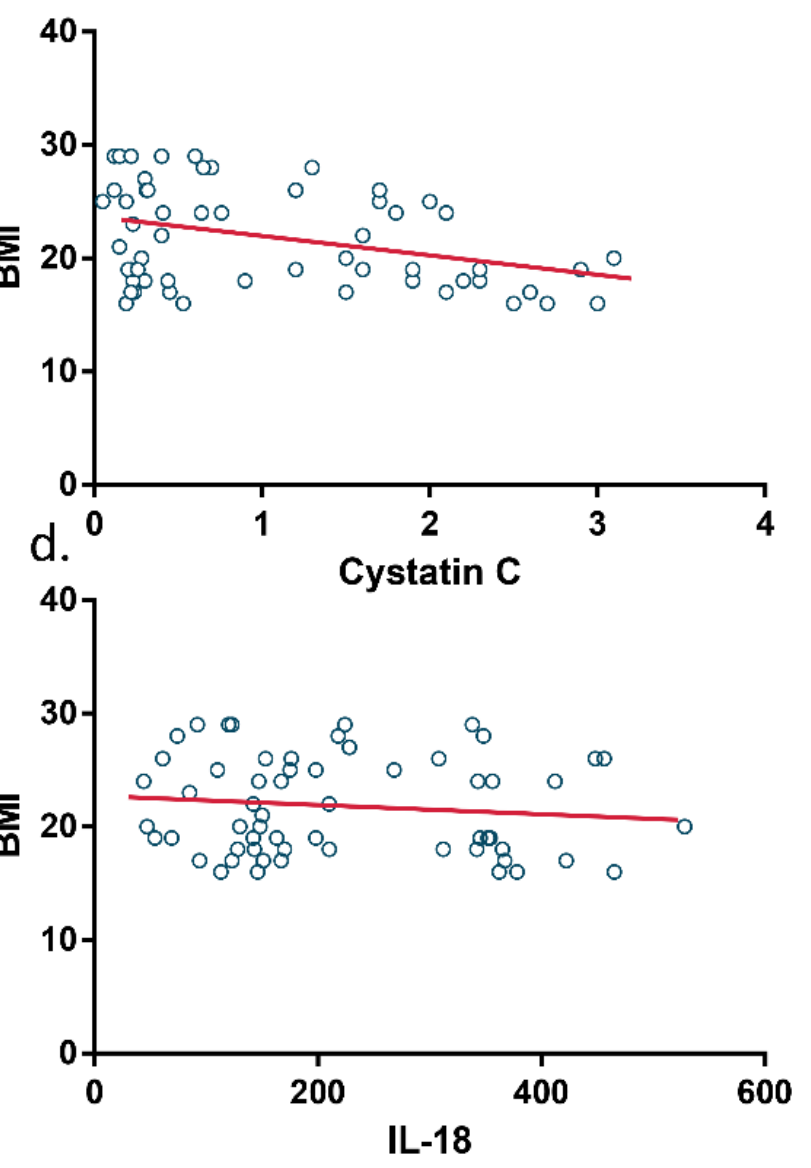

Figure (3): Scatter plot describing the negative correlation of renal biomarkers with age and body mass index (BMI). a; Cystatin C and age in months, b; Cystatin C and BMI, c; Interleukin 18 (IL-18) and age in months and d; IL-18 and BMI.

\section{DISCUSSION}

AKI is a life menacing hazard, especially in critically-ill children admitted to PICU. The insufficient evidence of biomarkers used in the early detection of AKI is the major hindrance in evolving newer preventive methods of AKI ${ }^{(13)}$. Whilst, serum creatinine and urea had limited evidence in early detection of $\mathrm{AKI}{ }^{(14)}$. Appropriate biomarkers must be excavated. Our study showed that both sCysC and sIL18 are the best biomarkers for prediction and early detection of AKI in critically-ill paediatric population.

Our result brought to light that; serum creatinine and urea was not authentic biomarkers in early diagnosis of AKI. On the other hand, sCys $\mathrm{C}$ and sIL18 exhibited higher diagnostic implementation of AKI even at the time of admission where they were increased dramatically with the progression of the disease on the third day with significantly higher levels in the case group in contrast with the control group.

In compliance with our results Lagos-Arevalo et al. ${ }^{(6)}$ notified that sCys $\mathrm{C}$ was optimal biomarker proved its ability in early diagnosis of AKI with high predictive value in AKI progression when obtained at the time of admission to PICU. Furthermore, serum Cys $\mathrm{C}$ reflected the intense drooping of GFR not only in children with AKI but also, in children with CKD with higher precision than serum creatinine ${ }^{(15)}$. Being not affected by the renal condition, nutritional status, and protein catabolism besides that its biochemical characters of free filtration in the glomeruli and the subsequent absorption in the proximal tubules make the CysC an ideal biomarker for assessing the GFR (16). Similarly, being a proinflammatory cytokine, this makes IL-18 an accurate biomarker for detection of the 
acute renal inflammation ${ }^{(17)}$. Despite many studies reported accuracy and superiority of both urine CysC and IL-18 over sCr ${ }^{(18-20)}$, serum levels of CysC and IL18 remain more accurate and superior than the urine level ${ }^{(17)}$. In AKI, serum level of both CysC and IL-18 rise before their urinary levels. For IL-18, it is expected for a cytokine to appear in the blood before the urine. $\mathrm{CysC}$, does not appear in the urine except after significant tubular injury and the tubular injury does not occur firstly in many cases of AKI ${ }^{(21)}$. Furthermore, a large proportion of the CysC that filtered through the glomerulus is metabolized by the kidney.

The restricted significance of $\mathrm{sCr}$ in the early detection of AKI may be attributed to the delayed increase in its level, especially after loss of $50 \%$ of the kidney function. Furthermore, sCr levels reverberate the glomerular function rather than the renal injury. Otherwise, sCr levels oftentimes rise in spite of the absence of internal kidney injury, whilst it may be normal when the damage is already occurred (22). Furthermore, its level is affected by several confounders as age, sex, hydration status and muscle bulk ${ }^{(2,5)}$. Moreover, the tubular fraction constitutes about $20 \%$ of the total excreted $\mathrm{Cr}$ which can mask the ameliorated GFR. In conformity with our results, SUN level was an improper early estimator of AKI nor prognosis of the kidney function because it is a lower airtight predictor of GFR ${ }^{(23)}$.

The strong negative correlations between both either sCysC or sIL-18 and age are concomitant with similar studies on neonates ${ }^{(18)}$ and adults ${ }^{(24)}$. However, we can attribute the negative association with BMI as the underweight or low-normal patients were more than the overweight or high-normal patients. This can explain the contradiction with other studies that illustrated the positive association between either sCysC or sIL-18 and BMI (24).

This was one hospital and limited universality study represented with a small sample population that restricted our ability to divide the patients into different groups and performing sub-group analysis. Also, the basal levels of IL-18 and CysC weren't estimated at the time of admission. Consequently, the actual timing of biomarker altitude in compliance with clinical sequelae cannot be assessed. Future studies should be implemented to detect the optimal rising time of IL-18 and $\mathrm{CysC}$ in comparison with the progression of $\mathrm{AKI}$. In conclusion, $\mathrm{sCysC}$ and serum interleukin-18 proved their accuracy in prediction and early detection of AKI in patients admitted to PICU and also for the follow up. Their diagnostic value was not only superior to serum creatinine and serum urea but also the urinary IL-18 and CysC.

\section{REFERENCES}

1. Flor RJ, Williams KN, O'Keeffe T (2018): Acute Kidney Injury. Surgical Critical Care and Emergency Surgery: Clinical Questions and Answers, onlinelibrary.wiley.com $>$ doi > abs

2. Momtaz HE, Sabzehei MK, Rasuli B, Torabian S (2014): The main etiologies of acute kidney injury in the newborns hospitalized in the neonatal intensive care unit. Journal of clinical neonatology, 3 (2): 99.

3. Mittal K, Aggarwal H, Mittal A (2007): Biomarkers: Acute Kidney Injury. Injury, 1: 4.

4. Edelstein CL (2017): Biomarkers in acute kidney injury. Biomarkers of Kidney Disease (Second Edition): Elsevier, Pp: 241-315.

5. Hewitt SM, Dear J, Star RA (2004): Discovery of protein biomarkers for renal diseases. Journal of the American Society of Nephrology, 15 (7): 1677-89.

6. Lagos-Arevalo P, Palijan A, Vertullo L, Devarajan P, Bennett MR, Sabbisetti V et al. (2015): Cystatin C in acute kidney injury diagnosis: early biomarker or alternative to serum creatinine? Pediatric Nephrology, 30 (4): 665-76.

7. Dinarello CA (2007): Interleukin-18 and the pathogenesis of inflammatory diseases.

www.ncbi.nlm.nih.gov > pubmed

8. Parikh CR, Devarajan P (2008): New biomarkers of acute kidney injury. Critical care medicine, 36 (4): S159S65.

9. Parikh C, Jani A, Mishra J, Ma Q, Kelly C, Barasch J et al. (2006): Urine NGAL and IL- 18 are predictive biomarkers for delayed graft function following kidney transplantation. American Journal of Transplantation, 6 (7): 1639-45.

10. Laterza OF, Price CP, Scott MG (2002): Cystatin C: an improved estimator of glomerular filtration rate? Clinical chemistry, 48 (5): 699-707.

11. Herget-Rosenthal S, Marggraf G, Hüsing J, Göring F, Pietruck F, Janssen $O$ et al. (2004): Early detection of acute renal failure by serum cystatin C. Kidney international, 66 (3): 1115-22.

12. Brion LP, Fleischman AR, McCarton C, Schwartz GJ (1986): A simple estimate of glomerular filtration rate in low birth weight infants during the first year of life: noninvasive assessment of body composition and growth. The Journal of pediatrics, 109 (4): 698-707.

13. Devarajan P (2008): Neutrophil gelatinase- associated lipocalin (NGAL): a new marker of kidney disease. Scandinavian journal of clinical and laboratory investigation, 68 (241): 89-94.

14. Schrezenmeier E, Barasch J, Budde K, Westhoff T, Schmidt-Ott K (2017): Biomarkers in acute kidney injury-pathophysiological basis and clinical performance. Acta physiologica, 219 (3): 556-74. 
15. Zappitelli M, Parvex P, Joseph L, Paradis G, Grey V, Lau $S$ et al. (2006): Derivation and validation of cystatin $\mathrm{C}$-based prediction equations for GFR in children. American journal of kidney diseases, 48 (2): 221-30.

16. Madero M, Sarnak MJ, Stevens LA (2006): Serum cystatin $\mathrm{C}$ as a marker of glomerular filtration rate. Current opinion in nephrology and hypertension, 15 (6): 610-6.

17. Leslie JA, Meldrum KK (2008): The role of interleukin18 in renal injury. Journal of Surgical Research, 145 (1): $170-5$.

18. Li Y, Fu C, Zhou X, Xiao Z, Zhu X, Jin M et al. (2012): Urine interleukin-18 and cystatin- $\mathrm{C}$ as biomarkers of acute kidney injury in critically ill neonates. Pediatric nephrology (Berlin, Germany), 27 (5): 851-60.

19. Lin $X$, Yuan $J$, Zhao $Y$, Zha $Y$ (2015): Urine interleukin-18 in prediction of acute kidney injury: a systemic review and meta-analysis. Journal of nephrology, 28 (1): 7-16.

20. Zhang Z, Lu B, Sheng $X$, Jin $\mathbf{N}$ (2011): Cystatin $C$ in prediction of acute kidney injury: a systemic review and meta-analysis. American Journal of Kidney Diseases, 58 (3): $356-65$.

21. Tenstad O, Roald AB, Grubb A, Aukland K (1996): Renal handling of radiolabelled human cystatin $\mathrm{C}$ in the rat. Scandinavian Journal of Clinical and Laboratory Investigation, 56: 409-14.

22. Parikh CR, Han G (2013): Variation in performance of kidney injury biomarkers due to cause of acute kidney injury. American Journal of Kidney Diseases, 62 (6): 1023-6.

23. Smith GL, Shlipak MG, Havranek EP, Foody JM, Masoudi FA, Rathore SS et al. (2006): Serum urea nitrogen, creatinine, and estimators of renal function: mortality in older patients with cardiovascular disease. Archives of internal medicine, 166 (10): 1134-42.

24. Stevens LA, Schmid CH, Greene T, Li L, Beck GJ, Joffe MM et al. (2009): Factors other than glomerular filtration rate affect serum cystatin $\mathrm{C}$ levels. Kidney international, 75 (6): 652-60. 